Wien klin Mag 2017 · 20:111

DOI 10.1007/s00740-017-0185-y

(c) Springer-Verlag GmbH Austria 2017

CrossMark

\author{
Verena Kienast \\ SpringerMedizin, Wien, Österreich
}

\title{
Angekommen bei sich selbst
}

\section{Abenteuer am Berg und im Alltag}

Eine ziemlich verrückte Idee: Einen Berg besteigen mit Transplantationspatienten. Und zwar nicht irgendeinen Berg, sondern den Kilimandscharo mit einer Gipfelhöhe von knapp 6000 Metern, wo die Luft schon ziemlich dünn ist und die Wetterverhältnisse nicht immer angenehm sind. Und nicht irgendwelche Transplantationspatienten, sondern ausgerechnet solche, die eine neue Lunge erhalten haben. Das - wohl kalkulierte und ausgezeichnet vorbereitete - Abenteuer, das der Pulmologe der MedUniWien Dr. Peter Jaksch initiierte und organisierte, war ein Erfolg: Nur zwei der insgesamt zehn Patienten mussten auf 4000 Metern Höhe umkehren, alle anderen erreichten den Gipfel.

Womit gezeigt werden konnte, dass solche Extremleistungen nach einem derart schweren Eingriff, wie einer Lungentransplantation, möglich sind. Natürlich waren die Teilnehmer extrem gut vorbereitet, sind durch regelmäßiges Training in sehr gutem Zustand, gehen auch regelmäßig Bergsteigen, nehmen teilweise an Marathons teil und haben, wie Jaksch berichtete, eine Lungenfunktion von 100 Prozent, waren damit also teilweise besser in Form als mancher der insgesamt 24 Begleiter, die sich aus Chirurgen, Pulmologen, Physiotherapeuten, usw. und Angehörigen zusammensetzte. Der Nutzen dieses Ereignisses geht dabei weit über den Werbeeffekt für das höchst erfolgreiche Lungentransplantationsprogramm an der Wiener Universitätsklinik hinaus, wo seit den Anfängen 1989 mehr als 1800 Transplantationen durchgeführt wurden.

\section{Erkenntnisse für die Wissenschaft und Motivation}

Zum einen wurde die Expedition auch wissenschaftlich begleitet, wurden unter anderem regelmäßig Blutgaswerte gemessen, ein Schlafscreening und Atemmuskeltests durchgeführt, einerseits um den Zustand der Patienten unter der extremen Belastung zu überprüfen und bei kontinuierlicher Verschlechterung auch entsprechend reagieren zu können. Diese Fülle an Werten wird nun aber in der Folge auch wissenschaftlich ausgewertet und neue Erkenntnisse über physiologische Veränderungen und Einflussfaktoren bei Menschen nach Transplantation in Extremsituationen sind dabei zu erwarten. Bis auf die zwei Patienten, die rechtzeitig umkehrten und deren Zustand sich in geringerer Höhe rasch besserte, hatte niemand mit schwerwiegenden gesundheitlichen Problemen zu kämpfen. Leichte Höhenkrankheit mit Übelkeit, Kopfschmerzen und Schlafstörungen ist in solchen Höhen durchaus verbreitet und betraf etwas mehr als die Hälfte der Teilnehmer - Patienten wie Begleitpersonen gleichermaßen.

Das Erfolgserlebnis am Gipfel war zweifellos für die früher schwerkranken Patienten mit nun neuer Lunge unbeschreiblich - und soll auch Motivation für andere Menschen sein, nicht aufzugeben, aber auch, an sich selbst zu arbeiten. Jedes einzelne Schicksal der Hauptakteure dieser Expedition ist eine Geschichte von Leid und nun großer Freude. So wie beispielsweise jener 62jährige Teilnehmer, der als 22-Jähriger den Gipfel des Nanga Parbat erreichte und nun - mit Spenderlunge - jenen des Klimandscharo.

\section{Ohne Allüren mit gegenseitigem Respekt}

Schließlich hat das Unternehmen „Mit neuer Lunge am Kilimandscharo" auch einen menschlich sehr verbindenden Aspekt unter den Teilnehmern: Ob Spitzenmediziner oder Patient, ob Angehöriger oder Medizintechniker - hier zählte die Kameradschaft und Unterstützung am Weg zum gemeinsamen Ziel. Ohne Allüren, ohne Wettbewerb, mit gegenseitigem Respekt und bedingungsloser Unterstützung. Ein wichtiger Impuls, der auch den Alltag in geringerer Höhe stärker prägen sollte, um das tägliche Abenteuer zu bewältigen. Denn Abenteuer bedeutet ankommen. Bei einem richtig bestandenen Abenteuer, so meinte einmal der Wiener Reiseschriftsteller, Photograph und Wanderer zwischen den Welten, Herbert Tichy, ist man angekommen bei sich selbst.

Das wünscht Ihnen Ihre

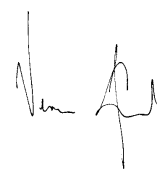

Verena Kienast

\section{Korrespondenzadresse}

V. Kienast

SpringerMedizin

Wien, Österreich

Verena.kienast@springer.at 